\title{
Espagne : encore une nouvelle réforme éducative
}

\author{
Juan Carlos González Faraco
}

Traducteur : Philippe Rabaté

\section{CpenEdition}

\section{Journals}

Édition électronique

URL : http://journals.openedition.org/ries/3455

DOI : 10.4000/ries.3455

ISSN : 2261-4265

Éditeur

Centre international d'études pédagogiques

Édition imprimée

Date de publication : 1 septembre 2013

Pagination : 16-21

ISBN : 978-2-85420-600-5

ISSN : $1254-4590$

\section{Référence électronique}

Juan Carlos González Faraco, «Espagne : encore une nouvelle réforme éducative », Revue

internationale d'éducation de Sèvres [En ligne], 63 I septembre 2013, mis en ligne le 01 septembre 2016, consulté le 30 avril 2019. URL : http://journals.openedition.org/ries/3455 ; DOI : 10.4000/ries.3455

Ce document a été généré automatiquement le 30 avril 2019

(c) Tous droits réservés 


\title{
Espagne : encore une nouvelle réforme éducative
}

\author{
Juan Carlos González Faraco
}

Traduction : Philippe Rabaté

\section{Les réformes éducatives et la rhétorique politique}

1 Depuis la création de l'état démocratique actuel, qui a pris forme avec la Constitution de 1978, se sont succédé en Espagne quatre réformes générales du système éducatif, auxquelles il convient d'ajouter une cascade de réformes partielles d'importance. Dans chaque cas, l'éducation s'est transformée en un champ de bataille idéologique et politique entre des adversaires irréconciliables et souvent intransigeants.

2 Ce phénomène plonge ses racines dans les origines du système éducatif espagnol. Presque à la manière d'une fatalité, chaque fois qu'un gouvernement entreprend une réforme éducative, les vieux conflits se renouvellent (le secteur public face au privé, l'État face à l'Église catholique, le laïc face au religieux...) tout en adoptant des formes différentes, alors que surgissent de nouveaux conflits selon les circonstances du moment. Mais l'affrontement politique, la division sociale et l'incapacité à parvenir à un accord réapparaissent systématiquement. La réforme éducative actuelle, défendue par le gouvernement conservateur du Partido Popular (PP), est une preuve plus qu'éloquente de cette «tradition ». À l'évidence, cette nouvelle réforme est intimement liée au contexte présent, national et international, mais il serait malaisé de saisir son envergure sans prendre en considération cette trajectoire historique marquée par un âpre conflit sans cesse renouvelé au sujet de l'éducation en Espagne.

3 Partout, il est fréquent que les gouvernements, partis et groupes sociaux influents choisissent l'éducation comme théâtre privilégié pour défendre leur identité idéologique et exhiber ainsi leurs divergences - moins visibles - sur le plan des politiques économiques. Aussi les réformes éducatives sont-elles régulièrement assorties de discours dans lesquels affleure ordinairement une certaine dose de rhétorique et de théâtralité. Par exemple, les groupes parlementaires d'opposition, avant même que la nouvelle loi ne 
soit définitivement approuvée, signent devant les caméras de télévision un document dans lequel ils s'engagent à abroger cette loi dès que l'un d'entre eux aura le pouvoir, et ce, bien que ce qui les différencie - socialistes, communistes, nationalistes - soit de notoriété publique. L'éducation fait assurément partie de la société du spectacle.

Dans les réformes récentes, l'on a pu apprécier l'hégémonie patente d'une vision avant tout économique de l'éducation. Des termes comme qualité, excellence, compétitivité, évaluation et d'autres, similaires, sont aujourd'hui communs à toutes les politiques éducatives mondiales. Ils apparaissent également dans cette réforme du gouvernement espagnol qui s'appuie sur une Loi organique précisément intitulée "Loi pour l'amélioration de la qualité éducative » (LOMCE). Au sens strict, cette loi ne modifie pas substantiellement la structure actuelle du système éducatif espagnol, établie en 1990 par le gouvernement socialiste de Felipe González. De fait, cette réforme a survécu au premier gouvernement conservateur (dont la loi de Qualité de l'éducation, de 2002, n'avait pas réussi à être appliquée), et elle a perduré, avec de légères adaptations, à travers la seconde réforme socialiste (Loi d'éducation de 2006).

Comme il fallait s'y attendre, après les élections de 2011, le nouveau ministre de l'éducation du PP a repris, du moins en partie, la loi précédente de 2002 pour lancer sa propre réforme de la dernière loi socialiste qui, sans bouleverser la structure du système, a modifié quelques éléments clés de son contenu. Le message martelé par le ministre, et reflété par le texte de la nouvelle loi, insiste avec des accents dramatiques sur l'échec du système éducatif espagnol, en s'appuyant principalement sur des données extraites d'une analyse comparée et internationale. La méthode employée consiste à tenter de justifier objectivement les décisions à prendre.

6 Néanmoins, la polémique enflammée que déclenche toute réforme éducative en Espagne a été à cette occasion accrue par la grave crise économique que vit le pays. Les réductions budgétaires considérables qui affectent, entre autres, le financement des collèges, le salaire des professeurs (parallèlement à l'augmentation de leurs heures de travail hebdomadaires), les bourses pour les étudiants universitaires, ne font pas partie, au sens propre, de cette réforme du système d'enseignement, mais en sont indissociables. Dans ce contexte, il n'est pas surprenant que le ministre de l'éducation se soit attiré les foudres des professeurs et étudiants et qu'il jouisse du plus bas niveau de popularité de tout le gouvernement. Cette ambiance crispée n'est assurément pas la plus propice à une analyse équilibrée des lignes directrices de cette réforme.

\section{Un diagnostic basé sur des rapports internationaux}

7 Il va de soi que l'Espagne n'est pas une exception face à l'influence croissante de l'internationalisation dans le champ de l'éducation. En se fondant sur des rapports de l'OCDE ou d'autres organisations internationales, en particulier européennes, la nouvelle loi indique quelques réussites du système éducatif mais elle souligne surtout ses faiblesses, par comparaison avec les données moyennes des pays de l'OCDE ou de l'Union européenne. À savoir :

- la faible proportion de jeunes diplômés de l'enseignement secondaire, notamment inférieur, conséquence, en grande partie, d'un abandon précoce du système par plus de $25 \%$ des élèves, et cause, du moins en partie, du taux excessivement élevé de chômage des jeunes $(57,2 \%)$; 
- la prédominance de l'enseignement général dans l'éducation secondaire supérieure ${ }^{1}$ face à la formation professionnelle ;

- les faibles résultats dans les épreuves d'évaluation de l'enquête Pisa, inférieurs à la moyenne des pays de l'OCDE.

comme, par exemple, la faible qualité de la formation et du modèle de recrutement du professorat de l'enseignement secondaire, les inégalités nées d'un double système publicprivé ou les grandes différences entre régions et communautés autonomes. En ce sens, on pourra comprendre que les nationalistes basques aient adopté une attitude de rejet face à cette réforme, entre autres raisons parce qu'ils la considèrent comme "superflue » pour le Pays Basque. Dans cette communauté autonome, outre le fait qu'elle jouit de taux de chômage inférieurs à la moyenne nationale, la dépense moyenne par élève et par an se situe bien au-dessus de la moyenne espagnole et atteint presque le double de celle de certaines régions du sud de la péninsule; le secteur privé, avec une forte présence de l'Église catholique, est légèrement supérieur au public (en Espagne, les établissements privés accueillent en moyenne $33 \%$ des élèves scolarisés) et les résultats des étudiants basques dans le cadre de l'enquête PISA sont supérieurs à la moyenne de l'OCDE. En effet, la décentralisation de l'éducation en Espagne, qui a apporté des améliorations indéniables au système scolaire, n'a pas permis de diminuer suffisamment les différences entre régions, affectant ainsi de manière négative l'égalité des chances de tous les citoyens et, probablement, la cohésion nationale. Cette situation expliquerait l'intérêt de la nouvelle loi qui offrirait l'opportunité au gouvernement de récupérer une partie du contrôle sur la politique éducative, tout particulièrement en ce qui concerne la définition du curriculum. À cet égard, la loi établit qu'au moins $50 \%$ de l'horaire d'enseignement doit correspondre à des matières communes à l'ensemble du territoire national et dont les contenus (parmi lesquels figure bien sûr la langue espagnole) seront établis par le gouvernement central. Les partis nationalistes sont très opposés à ce qu'ils considèrent comme une «recentralisation» de la politique éducative, bien que ce ne soit en réalité que partiellement le cas.

\section{Quel modèle d'éducation ?}

L'élément central de cette réforme est son orientation, c'est-à-dire son modèle d'éducation basé sur :

- une conception plus individuelle (l'effort, le talent, la diversité des capacités et attentes de chaque élève) que sociale du processus éducatif; d'où la diminution du degré de compréhensivité du système (axe central des réformes socialistes) par le biais de la diversification et de l'anticipation d'itinéraires scolaires distincts en fonction du succès de chaque élève lors des évaluations ;

- l'idée que l'éducation est une pièce maîtresse pour le développement économique, la mobilité sociale et l'emploi et que, par conséquent, les institutions scolaires doivent se soumettre à des critères de productivité et de reddition publique de comptes, fondamentalement à travers l'évaluation externe et standardisée des résultats d'apprentissage des élèves et la professionnalisation de la gestion des institutions ;

Revue internationale d'éducation de Sèvres, 63 | septembre 2013 
- la vision de l'éducation comme un droit de choisir de la part des familles, droit que l'État doit garantir, si bien que la demande familiale d'éducation se transforme en facteur déterminant pour l'assignation de la dépense éducative étatique. Dans la pratique, ceci suppose un appui explicite à l'enseignement privé sous contrat, autrement dit soutenu par des fonds publics, et, en ce qui concerne le curriculum, le renforcement de la religion comme matière soumise à évaluation à l'instar des autres (optionnelle pour les élèves mais proposée obligatoirement dans les écoles publiques, comme auparavant).

11 À l'évidence, en dépit de quelques particularités nationales, les lignes directrices de cette réforme ainsi que sa terminologie font partie de tendances internationales très répandues, et nombre des mesures qu'elle propose peuvent être observées dans d'autres pays. Les plus importantes concernent l'éducation secondaire obligatoire (de 12 à 16 ans) et post-obligatoire (16-18 ans), qui constituent, surtout pour la première, les périodes scolaires où l'on observe les données les plus problématiques et, dans certains cas, alarmantes : un tiers des jeunes Espagnols n'obtient pas le premier diplôme qu'offre le système éducatif ; ils échouent dans leur scolarité fondamentale et auront ainsi, comme on peut l'imaginer, de graves difficultés pour trouver un emploi et pour s'intégrer socialement de manière appropriée.

\section{Objectif central : l'éducation secondaire}

12 Pour faire face à cette situation, la réforme propose que les élèves choisissent des itinéraires alternatifs, en fonction de leurs résultats scolaires et de leurs intérêts personnels à partir de l'âge de 14-15 ans, soit durant le second cycle de l'éducation secondaire inférieure ou obligatoire. Pour éviter l'abandon précoce du système par ceux qui n'achèvent pas avec succès l'éducation secondaire, on leur offre des programmes de suivi spécifique et, surtout, une voie alternative, la " formation professionnelle de base » avec la possibilité d'une réintégration ultérieure dans le système général. Il existait déjà auparavant des options avec un objectif similaire bien qu'elles aient donné, pour la plupart d'entre elles, des résultats insatisfaisants. En tout état de cause, un des desseins essentiels de la réforme est de privilégier la formation professionnelle comme une voie éducative qui puisse favoriser l'emploi des jeunes.

L'évaluation des apprentissages par l'entremise d'épreuves nationales élaborées par le gouvernement central et inspirées des modèles internationaux comme, par exemple, Pisa, est l'une des mesures fondamentales que propose cette loi. Depuis la réforme de 1970 (dans les dernières années de la dictature franquiste), ce type d'évaluations avait disparu du système éducatif espagnol. Il est à présent de retour sous la forme d'un système de mesure des compétences des élèves et, partant, comme instrument d'orientation et de sélection de l'effectif scolaire mais également de classification et de mesure de la productivité des institutions éducatives. Par conséquent, comme un système de contrôle extérieur et standardisé. À l'évidence, la loi stipule qu'il s'agit d'un système pensé pour diagnostiquer les problèmes de rendement des élèves et des écoles et favoriser ainsi une amélioration de leur qualité. Les épreuves les plus importantes se situent à la fin de chaque étape éducative, mais celles qui sont véritablement décisives correspondent à l'éducation secondaire obligatoire ( 16 ans) et à l'éducation secondaire post-obligatoire (18 ans). 

secondaire. Outre le fait qu'elle s'efforce d'augmenter son uniformité à l'échelle nationale moyennant un contrôle accru des contenus de base et de leur évaluation de la part du gouvernement, l'intention de la réforme est de simplifier l'offre de matières et de privilégier ce que l'on appelle les apprentissages instrumentaux, fondamentalement la langue et les mathématiques. De cette manière, le curriculum demeure divisé en trois types de matières: celles qui relèvent du tronc commun ou fondamentales (avec les mêmes contenus sur tout le territoire national et qui occuperont au moins la moitié du temps scolaire), les matières spécifiques (à caractère optionnel et au contenu plus adaptable régionalement ou localement) et celles de libre configuration (par exemple, la langue propre de telle communauté autonome ou région).

Dans le cadre de cette réforme éducative, et comme dans tout processus de ce type, s'entremêlent des facteurs très divers : certains sont avant tout nationaux et proviennent de traditions historiques propres ou du contexte politique actuel; d'autres sont directement liés à la mondialisation de l'éducation si pertinemment étudiée par les professeurs Meyer et Ramírez ${ }^{2}$. Dans celle-ci comme dans d'autres réformes actuelles, l'on observe un déclin des principes qui avaient régi les politiques éducatives de la seconde moitié du XXe siècle, avec leurs déclarations en faveur de l'équité, souvent accompagnées d'une rhétorique de la rédemption sociale. Et l'on observe également la poussée apparemment impossible à contenir - de politiques de plus en plus assujetties à des critères méritocratiques, économiques et même financiers. Naturellement, il ne s'agit pas d'un jeu absolument nouveau ni d'un changement d'orientation soudain comme les partisans de l'un ou de l'autre bord politique veulent le faire croire : il s'agit seulement d'un pas supplémentaire dans une trajectoire perceptible depuis des années en Espagne et, bien sûr en Europe.

\section{NOTES}

1. L'enseignement secondaire comprend deux cycles distincts : quatre ans pour l'enseignement secondaire inférieur ou obligatoire (appelé «ESO ») et deux ans pour l'enseignement secondaire post-obligatoire ou baccalauréat (bachillerato). (NdT)

2. Meyer John W. \& Francisco O. Ramírez (2000). The World Institutionalization of Education: Origins and Implications. In Jürgen Schriewer (ed.) Discourse Formation in Comparative Education. Frankfurt: Peter Lang.

Meyer J.W. (2009). Reflections: Institutional Theory and World Society. In Georg Krücken \& Gili S. Drori (eds.) World Society: The Writings of John W. Meyer. Oxford: Oxford University Press.

Revue internationale d'éducation de Sèvres, 63 | septembre 2013 
INDEX

Mots-clés : enseignement secondaire, réforme de l'enseignement, système scolaire Index géographique : Espagne

\section{AUTEURS}

\section{JUAN CARLOS GONZÁLEZ FARACO}

Juan Carlos Gonzalez Faraco est professeur de théorie et d'histoire de l'éducation à l'Université de Huelva (Espagne). 